\title{
The Chance that a Convex Body Is Lattice-Point Free: A Relative of Buffon's Needle Problem
}

\author{
Imre Bárány ${ }^{1,2}$ \\ ${ }^{1}$ Rényi Institute of Mathematics, Hungarian Academy of Sciences, P.O. Box 127, \\ 1364 Budapest, Hungary; e-mail: barany@renyi.hu \\ ${ }^{2}$ Department of Mathematics, University College London, Gower Street, \\ London WC1E 6BT, England
}

Received 20 April 2005; accepted 29 December 2005

Published online 26 September 2006 in Wiley InterScience (www.interscience.wiley.com).

DOI 10.1002/rsa.20138

\begin{abstract}
Given a convex body $K \subset \mathbf{R}^{d}$, what is the probability that a randomly chosen congruent copy, $K^{*}$, of $K$ is lattice-point free, that is, $K^{*} \cap \mathbf{Z}^{d}=\emptyset$ ? Here $\mathbf{Z}^{d}$ is the usual lattice of integer points in $\mathbf{R}^{d}$. Luckily, the underlying probability is well defined since integer translations of $K$ can be factored out. The question came up in connection with integer programming. We explain what the answer is for convex bodies of large enough volume. (c) 2006 Wiley Periodicals, Inc. Random Struct. Alg., 30, 414-426, 2007
\end{abstract}

\section{INTRODUCTION}

Let $\mathbf{Z}^{d}$ denote the integer lattice in the $d$-dimensional Euclidean space $\mathbf{R}^{d}$. A random copy, $L$, of $\mathbf{Z}^{d}$ is just $L=L_{\rho, t}=\rho\left(\mathbf{Z}^{d}+t\right)$ where $t \in[0,1)^{d}$ is a translation vector and $\rho \in S O(d)$ is a rotation of $\mathbf{R}^{d}$ around the origin. We can, of course, replace $[0,1)^{d}$ by any other basis parallelotope of $\mathbf{Z}^{d}$. Setting

$$
\mathcal{L}=\left\{L_{\rho, t}: \rho \in S O(d), t \in[0,1)^{d}\right\},
$$

there is a probability measure Prob on $\mathcal{L}$, which is the product of the Lebesgue measure on $[0,1)^{d}$ and of the normalized Haar measure on $S O(d)$. The following question, which is a

Correspondence to: I. Bárány

(C) 2006 Wiley Periodicals, Inc. 
distant relative of Buffon's needle problem, emerged while investigating [2] the randomized integer convex hull, $I_{L}(K)=\operatorname{conv}(K \cap L)$ of a convex body $K \subset \mathbf{R}^{d}$. What is the probability that $K \cap L=\varnothing$ ? Note that in the abstract, the same question is formulated slightly differently.

This probability is clearly zero if $K$ is "large," for instance, if it contains a ball of radius $\sqrt{d} / 2$. But it is not zero if $K$ is "flat." We show first an upper bound for the probability in question. Let $\mathcal{K}^{d}$ denote the set of all convex bodies (i.e., convex compact sets with nonempty interior) in $\mathbf{R}^{d}$.

Theorem 1.1. For every $d \geq 2$ there exist positive constants $c_{1}(d)$ and $c_{2}(d)$ such that for every $K \in \mathcal{K}^{d}$ with $\operatorname{Vol} K \geq c_{2}(d)$,

$$
\operatorname{Prob}[K \cap L=\emptyset] \leq \frac{c_{1}(d)}{\operatorname{Vol} K} .
$$

Our next theorem shows that this result is the best possible apart from the constants $c_{i}$. We need a definition. Given a unit vector $t \in S^{d-1}$, the width of $K \in \mathcal{K}^{d}$ in direction $t$ is defined as

$$
w(K, t)=\max \{t(x-y): x, y \in K\},
$$

and the width, or geometric width of $K$ is

$$
w(K)=\min \left\{w(K, t): t \in S^{d-1}\right\} .
$$

Theorem 1.2. For every $d \geq 2$ there exist positive constants $b_{1}(d), b_{2}(d)$, and $w_{d}$ such that for every $K \in \mathcal{K}^{d}$ with $\operatorname{Vol} K \geq b_{2}(d)$ and $w(K) \leq w_{d}$

$$
\operatorname{Prob}[K \cap L=\emptyset] \geq \frac{b_{1}(d)}{\operatorname{Vol} K} .
$$

The constant $w_{d}$ is not too small: we can take it to be $1 /\left(2 d^{3 / 2}\right)$ for instance. What Theorems 1.1 and 1.2 state is that $\operatorname{Prob}[K \cap L=\emptyset]$ is of order $1 / \operatorname{Vol} K$ for convex bodies $K$ with large volume and $w(K) \leq w_{d}$. It is not clear (at least for the author) for which convex body of volume $V$ the probability in question is the largest.

Using Vinogradov « notation these results can be formulated more concisely as

$$
\operatorname{Prob}[K \cap L=\emptyset] \ll \frac{1}{\operatorname{Vol} K}
$$

for every $K \in \mathcal{K}^{d}$ of large volume and as

$$
\operatorname{Prob}[K \cap L=\emptyset] \gg \frac{1}{\operatorname{Vol} K}
$$

for every $K \in \mathcal{K}^{d}$ of large volume and small geometric width. Theorems 1.1 and 1.2 imply the following.

Corollary 1.3. For every $d \geq 2$, as $V \rightarrow \infty$,

$$
\frac{1}{V} \ll \sup \left\{\operatorname{Prob}[K \cap L=\emptyset]: K \in \mathcal{K}^{d}, \operatorname{Vol} K=V\right\} \ll \frac{1}{V} .
$$


The planar case of both Theorems is proved in [2]. So we assume, from now on, that $d \geq 3$. The paper is organized as follows. The next section explains the application of the above results for the randomized integer convex hull. In Section 3 notation, terminology, and some basic observations are described. Sections 4 and 5 contain the proofs of Theorems 1.1 and 1.2.

\section{APPLICATION: THE RANDOMIZED INTEGER CONVEX HULL}

For $K \in \mathcal{K}^{d}$ define the function $u: K \rightarrow \mathbf{R}$ by

$$
u(x)=\operatorname{Vol}(K \cap(x-K))
$$

that is, $u(x)$ is the volume of the so-called Macbeath region, which is the intersection of $K$ with $K$ reflected around the point $x \in K$. Information on properties of the Macbeath region and $u(x)$ is available in $[3,6,10]$ or $[1]$. We also set

$$
K(u \leq t)=\{x \in K: u(x) \leq t\} .
$$

For $D>1$ define $\mathcal{K}_{D}=\mathcal{K}_{D}^{d}$ as the set of all $K \in \mathcal{K}^{d}$ for which $R / r \leq D$, where $R$ and $r$ denote the radii of the circumscribed and inscribed ball of $K$. In [2] we showed that the expected number, $E\left(f_{0}\left(I_{L}(K)\right)\right.$, of vertices of the randomized integer convex hull of a $K \in \mathcal{K}_{d}$ satisfies

$$
\operatorname{Vol} K(u \leq 1) \ll E\left(f_{0}\left(I_{L}(K)\right) \ll \operatorname{Vol} K(u \leq 1)\right.
$$

as Vol $K$ goes to infinity. It is known, see [3] for instance, that

$$
(\log \operatorname{Vol} K)^{d-1} \ll \operatorname{Vol} K(u \leq 1) \ll(\operatorname{Vol} K)^{(d-1) /(d+1)},
$$

where the implied constants depend only on $d$. Moreover, these estimates are best possible: the lower bound is reached for polytopes and the upper bound for smooth convex bodies.

Given $K \in \mathcal{K}_{d}$ and $L \in \mathcal{L}$, the missed volume is

$$
M(K, L)=\operatorname{Vol}\left(K \backslash I_{L}(K)\right)
$$

The expected missed volume is then the expectation of $M(K, L)$ over $L \in \mathcal{L}$ :

$$
M(K):=E M(K, L)
$$

We proved in [2] that, for $K \in \mathcal{K}_{D}$ in the planar case

$$
\int_{K} \frac{d x}{1+u(x)} \ll M(K) \ll \int_{K} \frac{d x}{1+u(x)} .
$$

For $d \geq 3$ Theorems 1.1 and 1.2 provide an identical upper bound and a weaker lower bound for $M(K)$. To state the results we introduce some new terminology. The function $v: K \rightarrow \mathbf{R}$ is defined as

$$
v(x)=\min \{\operatorname{Vol} K \cap H: x \in H, H \text { is a halfspace }\} .
$$


Given $x \in K$ the set $C(x)=K \cap H$ is a minimal cap if $H$ is a halfspace, $x \in H$, and $\operatorname{Vol} K \cap H=v(x)$. Assume $t \in S^{d-1}$ is the unit normal vector of the bounding hyperplane of $H$. We write $w(x)$ for the width of $C(x)$ in the direction of $t$ :

$$
w(x)=w(C(x), t)=\max \{t(y-z): y, z \in C(x)\} .
$$

The minimal cap of $x$ need not be unique, in which case let $w(x)$ be the supremum of the widths of the minimal caps of $x$. Finally, for $K \in \mathcal{K}_{D}$ write $K_{0}$ for the set of those $x \in K$ for which $w(x) \leq w_{d}$ where $w_{d}$ comes from Theorem 1.2.

Theorem 2.1. If $d \geq 2$ and $D>1$ and $K \in \mathcal{K}_{D}$ with $\operatorname{Vol} K \rightarrow \infty$, then

$$
\int_{K_{0} \cap K(u \geq 1)} \frac{d x}{u(x)} \ll M(K) \ll \int_{K} \frac{d x}{1+u(x)},
$$

where the constants implied by the $\ll$ notation depend only on $d$ and $D$.

Most likely, the upper and lower bounds are of the same order for every $K \in \mathcal{K}_{D}$. This is known for $d=2$ but the proof (see [2]) is very technical. Yet using this theorem one can determine the order of magnitude of $M(K)$ for smooth convex bodies,

$$
(\operatorname{Vol} K)^{(d-1) /(d+1)} \ll M(K) \ll(\operatorname{Vol} K)^{(d-1) /(d+1)},
$$

and for polytopes,

$$
(\log \operatorname{Vol} K)^{d} \ll M(K) \ll(\log \operatorname{Vol} K)^{d} .
$$

In both cases the implied constants depend on $K$ as well. The proofs of Theorem 2.1 and of the inequalities just stated follow those in [2] and are omitted.

\section{PREPARATIONS}

For $u \in \mathbf{R}^{d}, u \neq 0$ and $v>0$ define

$$
S(u, v)=\left\{x \in \mathbf{R}^{d}-v \leq u x \leq v\right\},
$$

which is just a slab orthogonal to $u$ and of width $2 v /|u|$. Here $|u|$ stands for the Euclidean norm of the vector $u \in \mathbf{R}^{d}$. Given a vector $a=\left(a_{1}, \ldots, a_{d}\right)$ in $\mathbf{R}^{d}$ with all $a_{i}>0$ we define

$$
\operatorname{Oct}(a)=\operatorname{conv}\left\{ \pm a_{1} e_{1}, \cdots \pm a_{d} e_{d}\right\}
$$

where $e_{1}, \ldots, e_{d}$ is the standard basis of $\mathbf{R}^{d}$. Clearly, $\operatorname{Oct}(a)$ is the octahedron with half-axes $a_{i}$ in direction $e_{i}$.

The Löwner-John theorem (see [5]) states that, given a convex body $K$ in $\mathbf{R}^{d}$, there is a pair ( $E, E^{\prime}$ ) of ellipsoids such that $E \subset K \subset E^{\prime}, E$ and $E^{\prime}$ are concentric, and $E$ arises from $E^{\prime}$ by shrinking by a factor of $1 / d$. We will need a similar result with octahedra replacing the ellipsoids:

Lemma 3.1. Given a convex body $K$ in $\mathbf{R}^{d}$, there is a positive vector a $\in \mathbf{R}^{d}$ such that a congruent copy, $K^{*}$, of $K$ satisfies

$$
\operatorname{Oct}(a) \subset K^{*} \subset \operatorname{Oct}\left(d^{3 / 2} a\right) .
$$


Proof. Let $\left(E, E^{\prime}\right)$ be the Löwner-John ellipsoid pair for $K$; let $a_{1} \leq a_{2} \leq \cdots \leq a_{d}$ denote the lengths of the half axes of $E$. Then the ellipsoid $\sum_{1}^{d}\left(x_{i} / a_{i}\right)^{2} \leq 1$ contains a congruent copy, $K^{*}$, of $K$. It is trivial to check that $\operatorname{Oct}(a) \subset K^{*} \subset \operatorname{Oct}\left(d^{3 / 2} a\right)$. We remark that $2 a_{1} \leq w(K)$ since the width of $E$ (which is $2 a_{1}$ ) is at most the width of $K$ because $E \subset K$.

A random element $\rho \in S O(d)$ takes a fixed orthonormal basis $b_{1}, \ldots, b_{d}$ of $\mathbf{R}^{d}$ to another orthonormal basis $\rho b_{1}, \ldots, \rho b_{d}$. For simpler notation we write $[d]=\{1,2, \ldots, d\}$ and we let $\lambda$ denote the usual rotation invariant $(d-1)$ dimensional measure on $S^{d-1}$ normalized so that $\lambda\left(S^{d-1}\right)=1$. It will be convenient to denote by $\operatorname{Prob}_{\rho}$ the normalized Haar measure on $S O(d)$ since it is a probability measure and we often want to talk about the probability of an event.

\section{Lemma 3.2. Under the above conditions,}

$$
\operatorname{Prob}_{\rho}[\operatorname{Oct}(a) \subset \rho S(u, v)]=\lambda\left\{f \in S^{d-1}:\left|f_{i}\right| \leq \frac{v}{a_{i}|u|} \forall i \in[d]\right\} .
$$

Proof. Fix an orthonormal basis $b_{1}, \ldots, b_{d}$ with $b_{1}=u /|u|$ and let $\rho b_{1}=f=\left(f_{1}, \ldots, f_{d}\right)$. Then $\rho S(u, v)=S(f, v /|u|)$. Here $S(f, v /|u|)$ contains $\operatorname{Oct}(a)$ if and only if

$$
\pm a_{i} e_{i} \in S(f, v /|u|) \forall i \in[d] .
$$

This is the same as $\left|a_{i} e_{i} f\right|=a_{i}\left|f_{i}\right| \leq v /|u|$.

As $f$ is a unit vector the probability in the lemma is positive if and only if

$$
1=\sum_{1}^{d} f_{i}^{2}<\sum v^{2} /\left(a_{i}^{2}|u|^{2}\right) .
$$

This condition is equivalent to $|u|^{2} / v^{2}<\sum a_{i}^{-2}$, which implies that if the probability in the Lemma is positive, then some $a_{i}$ must be "small."

Let us consider a vector $\alpha=\left(\alpha_{1}, \ldots, \alpha_{d}\right) \in \mathbf{R}^{d}$ such that $\alpha_{i}>0$ for all $i \in[d]$ and $\alpha_{i}>1$ for at least one $i \in[d]$. In this case,

$$
A=\left\{f \in S^{d-1}:\left|f_{i}\right| \leq \alpha_{i} \forall i \in[d]\right\}
$$

is nonempty. We have the following estimates.

Lemma 3.3. With the above notation,

$$
\prod_{i: \alpha_{i}<1} \alpha_{i} \ll \lambda(A) \ll \prod_{i: \alpha_{i}<1} \alpha_{i}
$$

Proof. We only give a sketch of the proof, which goes by induction on $d$. The case $d=2$ is clear. For the case $d-1 \rightarrow d$, assume that $\alpha_{d}$ is the smallest component of $\alpha$ and define $\alpha^{*}=\left(\alpha_{1}, \ldots, \alpha_{d-1}\right)$ and write $A^{*}$ for the corresponding set in $S^{d-2}$. The induction hypothesis can be used for $A^{*}$. Simple arguments finish the proof; details are left to the reader. 
The lattice width $W(K)$ of a convex body $K \in \mathcal{K}$ is, by definition,

$$
W(K)=\min _{z \in \mathbf{Z}^{d}, z \neq 0} \max \{z(x-y): x, y \in K\} .
$$

If the minimum is reached on $z \in \mathbf{Z}^{d}$, then $z$ is called the lattice width direction of $K$. Clearly, such a $z$ is a primitive vector, that is, the g.c.d. of the components of $z$ is 1 . We shall denote by $\mathbf{P}$ the set of all primitive vectors in $\mathbf{Z}^{d}$. Note that $0 \notin \mathbf{P}$. We will need the so-called Flatness Theorem, which is due to Khintchine [9], cf. [8] as well.

Theorem 3.4 (Flatness Theorem). If $C \in \mathcal{K}^{d}$ and $C \cap \mathbf{Z}^{d}=\emptyset$, then $W(C) \leq W_{d}$, where $W_{d}$ is a constant depending only on $d$.

\section{PROOF OF THEOREM 1.1}

Assume $K \in \mathcal{K}^{d}$ with Vol $K=V$ large. Lemma 3.1 implies the existence of an $a=$ $\left(a_{1}, \ldots, a_{d}\right) \in \mathbf{R}^{d}$ with $0<a_{1} \leq a_{2} \leq \cdots \leq a_{d}$ such that $V \ll \prod_{1}^{d} a_{i}$ and such that a congruent copy, $K^{*}$, of $K$ contains $\operatorname{Oct}(a)$. Here we may and do assume that

$$
a_{1} \leq \frac{a_{2}}{2} \leq \cdots \leq \frac{a_{d}}{2^{d-1}} .
$$

This can be achieved by keeping $a_{d}$ the same and replacing $a_{i}$ by $a_{i+1} / 2$ if $a_{i}>a_{i+1} / 2$ recursively for $i=d-1, d-2, \ldots, 1$. Clearly, this does not influence the validity of $V \ll \prod_{1}^{d} a_{i}$.

Now we begin the proof. First

$$
\operatorname{Prob}[K \cap L=\emptyset]=\operatorname{Prob}\left[K^{*} \cap L=\emptyset\right] \leq \operatorname{Prob}[\operatorname{Oct}(a) \cap L=\emptyset] .
$$

By the Flatness Theorem, Oct $(a) \cap L=\varnothing$ implies that the lattice width (in the lattice $L)$ of $\operatorname{Oct}(a)$ is at most $W_{d}$, which implies, in turn, that $\operatorname{Oct}(a) \subset \rho S\left(u, W_{d} / 2\right)$ for some $\rho \in S O(d)$ with suitable $u \in \mathbf{P}$, that is,

$$
\operatorname{Prob}[\operatorname{Oct}(a) \cap L=\emptyset] \leq \sum_{u \in \mathbf{P}} \operatorname{Prob}_{\rho}\left[\operatorname{Oct}(a) \subset \rho S\left(u, W_{d} / 2\right)\right] .
$$

The geometric width of $\operatorname{Oct}(a)$ is

$$
2\left(\sum_{1}^{d} \frac{1}{a_{i}^{2}}\right)^{-1 / 2} \geq 2\left(\sum_{i=1}^{d} \frac{1}{\left(2^{i-1} a_{1}\right)^{2}}\right)^{-1 / 2}>a_{1} \sqrt{3} .
$$

Since $\rho S\left(u, W_{d} / 2\right)$ cannot contain a set of width larger than $W_{d} /|u|$, we have

$$
a_{1} \sqrt{3}<\frac{W_{d}}{|u|}
$$

In other words, the sum over $u \in \mathbf{P}$ is to be restricted to $u$ with $|u| \leq \frac{W_{d}}{a_{1} \sqrt{3}}$. Let $\mathbf{P}^{*}$ denote the set of these $u \in \mathbf{P}$. 
Given such a $u \in \mathbf{P}^{*}$, let $i=i(u)$ be the smallest index $j$ with

$$
\frac{W_{d}}{a_{j}|u| \sqrt{3}}<1
$$

We have seen that $i(u)>1$. Thus, using Lemmas 3.2 and 3.3, we get for a fixed $u \in \mathbf{P}^{*}$ that

$$
\begin{aligned}
\operatorname{Prob}\left[\operatorname{Oct}(a) \subset \rho S\left(u, W_{d} / 2\right)\right] & =\lambda\left\{f \in S^{d-1}:\left|f_{j}\right| \leq \frac{W_{d}}{2 a_{j}|u|}, j \in[d]\right\} \\
& \ll \prod_{j=i(u)}^{d} \frac{W_{d}}{2 a_{j}|u|} \ll \prod_{j=2}^{d} \frac{1}{2 a_{j}|u|} \\
& \ll \frac{|u|^{-(d-1)}}{a_{2} \cdots a_{d}} .
\end{aligned}
$$

This shows that

$$
\sum_{u \in \mathbf{P}^{*}} \operatorname{Prob}\left[\operatorname{Oct}(a) \subset \rho S\left(u, W_{d} / 2\right)\right] \ll \frac{1}{a_{2} \ldots a_{d}} \sum_{u \in \mathbf{P}^{*}}|u|^{-(d-1)} .
$$

The last sum can be estimated from above by standard methods: instead of summing over $u \in \mathbf{P}^{*}$, we can sum over all $u \in \mathbf{Z}^{d} \cap B$ where $B$ is the ball centered at the origin and having radius $\frac{W_{d}}{a_{1} \sqrt{3}}$. This sum, in turn, differs little from the integral $\int_{B}|x|^{-d+1} d x$. Thus, we have

$$
\sum_{u \in \mathbf{P}^{*}}|u|^{-(d-1)} \leq \sum_{u \in \mathbf{Z}^{d} \cap B}|u|^{-(d-1)} \ll \int_{B}|x|^{-d+1} d x \ll \frac{1}{a_{1}} .
$$

This implies now that

$$
\sum_{u \in \mathbf{P}^{*}} \operatorname{Prob}\left[\operatorname{Oct}(a) \subset \rho S\left(u, W_{d} / 2\right)\right] \ll \frac{1}{a_{1} \ldots a_{d}} \ll \frac{1}{V} .
$$

\section{PROOF OF THEOREM 1.2}

This proof is more difficult than the previous one. We first show that it is enough to prove the theorem when $K$ is an octahedron: Lemma 3.1 implies that for every $K \in \mathcal{K}^{d}$ with Vol $K=V$ large there is $a=\left(a_{1}, \ldots, a_{d}\right) \in \mathbf{R}^{d}$ with $0<a_{1} \leq \cdots \leq a_{d}$ with $\prod a_{i} \ll V$ such that a congruent copy, $K^{*}$, of $K$ is contained in $\operatorname{Oct}(a)$. (The $a_{i}$ here are equal to what was $d^{3 / 2} a_{i}$ in Lemma 3.1.) It follows from the remark at the end of the proof of Lemma 3.1 that $2 a_{1} \leq d^{3 / 2} w(K)$. We may assume, again, that

$$
0<a_{1} \leq \frac{a_{2}}{2} \leq \cdots \leq \frac{a_{d}}{2^{d-1}}
$$

by keeping $a_{1}$ the same and replacing, recursively, $a_{i+1}$ by $2 a_{i}$ if $a_{i+1}<2 a_{i}$. It is clear that

$$
\operatorname{Prob}[K \cap L=\emptyset]=\operatorname{Prob}\left[K^{*} \cap L=\emptyset\right] \geq \operatorname{Prob}[\operatorname{Oct}(a) \cap L=\emptyset] .
$$


Set $\delta=0.48$. For fixed $u \in \mathbf{P}$ we define

$$
E(u)=\{\rho \in S O(d): \operatorname{Oct}(a) \subset \rho S(u, \delta)\}
$$

The slab $S(u, \delta)$ is a little smaller than the slab between two consecutive lattice hyperplanes orthogonal to $u$. This fact allows us to get rid of translations:

Claim 5.1. If $\rho \in E(u)$, then a positive fraction of all translations $t \in[0,1)^{d}$ have the property that Oct $(a)$ is between two consecutive lattice hyperplanes, orthogonal to $\rho u$, in the lattice $L=\rho\left(\mathbf{Z}^{d}+t\right)$.

Proof. Of course we can consider all translations $t \in B$ for an arbitrary basis parallelotope $B$ of $\mathbf{Z}^{d}$, not only for $B=[0,1)^{d}$. We choose $B$ so that the associated basis contains $u$. As $\operatorname{Oct}(a) \subset \rho S(u, \delta), \operatorname{Oct}(a)$ lies between two consecutive $L$-lattice hyperplanes orthogonal to $\rho u$ for at least $4 \%$ (as $2 \delta=0.96$ ) of translations $t \in B$ because only the $u$-component of $t$ matters.

We want to estimate, from below, the measure of $\bigcup_{u \in \mathbf{P}} E(u) \subset S O(d)$. Setting first

$$
\mathbf{P}^{*}=\left\{u \in \mathbf{P}: 2.1 \leq \frac{1}{a_{1}|u|} \leq 2.3\right\}
$$

and

$$
\mathbf{P}(u)=\left\{v \in \mathbf{P}^{*}:|v| \geq|u|, v \neq u\right\},
$$

we have

$$
\begin{aligned}
\operatorname{Prob}_{\rho}\left[\bigcup_{u \in \mathbf{P}} E(u)\right] & \geq \operatorname{Prob}_{\rho}\left[\bigcup_{u \in \mathbf{P}^{*}} E(u)\right] \\
& \geq \sum_{u \in \mathbf{P}^{*}}\left(\operatorname{Prob}_{\rho}[E(u)]-\sum_{v \in \mathbf{P}(u)} \operatorname{Prob}_{\rho}[E(u) \cap E(v)]\right) .
\end{aligned}
$$

Our next target is to prove that $\sum_{u \in \mathbf{P}^{*}} \operatorname{Prob}_{\rho}[E(u)] \ll 1 / V$ and that $\sum_{u \in \mathbf{P}^{*}} \sum_{v \in \mathbf{P}(u)}$ $\operatorname{Prob}_{\rho}[E(u) \cap E(v)]$ is much smaller than $1 / V$.

Remark 1. We need the condition $w(K) \leq w_{d}$ since we need to have some nonempty $E(u)$. So we need some $u \in \mathbf{P}$ such that $\rho S(u, \delta)$ contains $\operatorname{Oct}(a)$, that is, $a_{1}$ must be smaller than $\delta /|u|$ for some $u \in \mathbf{P}$. As we have seen, $2 a_{1} \leq d^{3 / 2} w(K)$, we can take $w_{d}=1 /\left(2 d^{3 / 2}\right)$ implying $a_{1} \leq 1 / 4$. With this choice there are several primitive vectors satisfying the requirement.

Remark 2. We mention in passing that in the planar case there is no $\rho$ in $E(u) \cap E(v)$ since the intersection of the two slabs has area less than 1 and so it cannot contain $\operatorname{Oct}(a)$ or $K$. 
We continue with the proof. By the choice of $\mathbf{P}^{*}, \frac{\delta}{a_{1}|u|} \geq \delta \cdot 2.1>1$ and also $\frac{\delta}{a_{2}|u|}<1$ and we have, using Lemmas 3.2 and 3.3 again,

$$
\begin{aligned}
\sum_{u \in \mathbf{P}^{*}} \operatorname{Prob}_{\rho}[E(u)] & =\sum_{u \in \mathbf{P}^{*}} \lambda\left\{f \in S^{d-1}:\left|f_{j}\right| \leq \frac{\delta}{a_{j}|u|}, j \in[d]\right\} \\
& \gg \sum_{u \in \mathbf{P}^{*}} \prod_{j=2}^{d} \frac{\delta}{a_{j}|u|} \gg \sum_{u \in \mathbf{P}^{*}} \frac{|u|^{-(d-1)}}{a_{2} \ldots a_{d}} \\
& \gg \frac{1}{a_{2} \ldots a_{d}} \sum_{u \in \mathbf{P}^{*}}|u|^{-(d-1)}
\end{aligned}
$$

The last sum can be estimated from below by the standard method, which uses the Möbius function $\mu(d)$ (see, for instance, [7] page 268, or [4], Lemma 1, for very similar computations):

$$
\sum_{u \in \mathbf{P}^{*}}|u|^{-(d-1)} \gg \frac{1}{a_{1}} .
$$

We omit the routine details.

So we get that

$$
\sum_{u \in \mathbf{P}^{*}} \operatorname{Prob}_{\rho}[E(u)] \gg \frac{1}{V} .
$$

Our next target is to give an upper bound on $\sum_{v \in \mathbf{P}(u)} \operatorname{Prob}_{\rho}[E(u) \cap E(v)]$ when $u \in \mathbf{P}^{*}$ is fixed. This will be done in several steps.

Assume $\rho \in E(u) \cap E(v)$ and let $A$ be the two-dimensional plane spanned by $u$ and $v$. Further, let $\gamma$ denote the smaller angle between the lines of $u$ and $v$. Fix an orthonormal basis $b_{1}, b_{2}, \ldots, b_{d}$ with $b_{1}=u /|u|$ and $b_{2} \in A$, the rest of the $b_{i}$ arbitrary. (Of course $b_{1} \perp b_{2}$.) Suppose $\rho b_{1}=f$ and $\rho b_{2}=g$. Since $\operatorname{Oct}(a)$ lies in both $\rho S(u, \delta)$ and $\rho S(v, \delta)$, its projection onto $A$ lies in the parallelogram in Fig. 1.

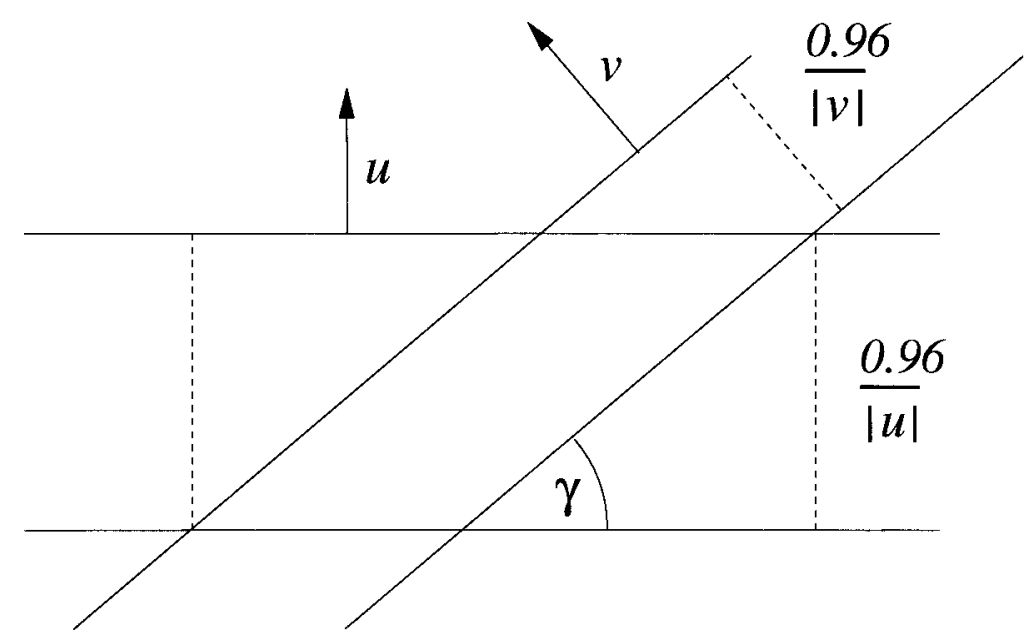

Fig. 1. The parallelogram in $A$. 
The radius of the ball inscribed to the $(d-1)$-dimensional octahedron $\operatorname{Oct}\left(a_{2}, \ldots, a_{d}\right)$ is

$$
\left(\sum_{2}^{d} \frac{1}{a_{i}^{2}}\right)^{-1 / 2} \geq a_{2} \sqrt{3}
$$

Thus, the diameter of the parallelogram in Fig. 1 is at least $2 a_{2} \sqrt{3}$, implying

$$
2 \sqrt{3} a_{2}<\frac{2 \delta}{\sin \gamma}\left(\frac{1}{|u|}+\frac{1}{|v|}\right) \leq \frac{4 \delta}{|u| \sin \gamma},
$$

and hence

$$
\sin \gamma<\frac{2 \delta}{\sqrt{3} a_{2}|u|} \leq \frac{2 \delta}{2 \sqrt{3} a_{1}|u|}<0.64
$$

The octahedron $\operatorname{Oct}(a)$ lies in the slab $\rho S(u, \delta) \subset S(f, \delta /|u|)$ and also in the slab $\rho S(v, \delta) \subset S(g, 2 \delta /|u| \sin \gamma)$, where $2 \delta /|u| \sin \gamma$ comes from the fact that the width (in direction $g$ ) of the parallelogram in Fig. 1 is at most $4 \delta /|u| \sin \gamma$, see (1). So we need to have

$$
\left|f_{i}\right| \leq \frac{\delta}{a_{i}|u|}=: \alpha_{i} \forall i \in[d], \text { and }\left|g_{i}\right| \leq \frac{2 \delta}{a_{i}|u| \sin \gamma}=: \beta_{i} \forall i \in[d] .
$$

Note that for $i=1$ both inequalities are satisfied.

Claim 5.2. If $f \in S^{d-1}$ and $\left|f_{i}\right| \leq \alpha_{i}$ for $i=2,3, \ldots, d$, then $\left|f_{1}\right| \geq 1 / \sqrt{2}$. Further, if $g \in S^{d-1}$ and $f \perp g$, then $\left|g_{1}\right|<1 / \sqrt{2}$.

Proof. This is simple:

$$
\begin{aligned}
\sum_{2}^{d} f_{i}^{2} & \leq \sum_{2}^{d} \alpha_{i}^{2} \leq \frac{\delta^{2}}{|u|^{2}}\left(\frac{1}{a_{2}^{2}}+\frac{1}{\left(2 a_{2}\right)^{2}}+\ldots\right) \\
& <\frac{\delta^{2} \cdot 4}{3|u|^{2} a_{2}^{2}} \leq \frac{\delta^{2}}{3|u|^{2} a_{1}^{2}}<\frac{\delta^{2} \cdot 2.3^{2}}{3}<\frac{1}{2} .
\end{aligned}
$$

(Here the last but one inequality follows from the definition: $u \in \mathbf{P}^{*}$ if and only if $\frac{1}{a_{1}|u|}$ lies in [2.1,2.3].) This implies the first part of the claim since $f$ is a unit vector. For the second part, assume $\left|g_{1}\right| \geq 1 / \sqrt{2}$. Then $\sum_{2}^{d} g_{i}^{2} \leq 1 / 2$ and since $\sum_{2}^{d} f_{i}^{2}<1 / 2$, the Cauchy-Schwarz inequality gives $\left|\sum_{2}^{d} f_{i} g_{i}\right|<1 / 2$ and we can't have $f \perp g$.

Now we return to estimating

$$
\operatorname{Prob}_{\rho}[E(u) \cap E(v)] \leq \lambda\left\{(f, g) \in S^{d-1} \times S^{d-1} f \perp g, \text { satisfying (2) }\right\} .
$$

For fixed $f$ define $G_{f}=\left\{g \in S^{d-1}: g \perp f,\left|g_{i}\right| \leq \beta_{i}, i=2, \ldots, d\right\}$ and $G_{f}^{*}=\{t g: g \in$ $\left.G_{f}, t \in[0,1]\right\}$. Let pr be projection from $\mathbf{R}^{d}$ onto the hyperplane $\left\{x \in \mathbf{R}^{d}: x_{1}=0\right\}$. $G_{f}$ lies on a $(d-2)$-dimensional great circle of $S^{d-1}$ and it is clear that

$$
\operatorname{Vol}_{d-2} G_{f}=(d-1) \operatorname{Vol}_{d-1} G_{f}^{*}=\frac{d-1}{\left|f_{1}\right|} \operatorname{Vol}_{d-1} \operatorname{pr} G_{f}^{*} .
$$


Now define the set $H=H(u, \gamma) \subset \mathbf{R}^{d-1}$ by

$$
H=\left\{h \in S^{d-2}:\left|h_{i}\right| \leq \sqrt{2} \beta_{i}, i=2, \ldots, d\right\}
$$

and $H^{*}=\{t h: h \in H, t \in[0,1]\}$. As we have seen, $g \in G_{f}$ implies $\left|g_{1}\right|<1 / \sqrt{2}$. Then $|\operatorname{pr} g|>1 / \sqrt{2}$ follows, showing that for each $g \in G_{f}$ the projection of the segment $[0, g]$ lies in $H^{*}$. In other words $\operatorname{pr} G_{f}^{*} \subset H^{*}$. Further, it is evident that

$$
(d-1) \operatorname{Vol}_{d-1} H^{*}=\operatorname{Vol}_{d-2} H .
$$

So we have

$$
\operatorname{Vol}_{d-2} G_{f} \leq \frac{1}{\left|f_{1}\right|} \operatorname{Vol}_{d-2} H \leq \sqrt{2} \operatorname{Vol}_{d-2} H
$$

Thus, we have, using Lemma 3.2,

$$
\begin{aligned}
\operatorname{Prob}_{\rho}[E(u) \cap E(v)] & \leq \lambda\left\{f \in S^{d-1}:\left|f_{i}\right| \leq \alpha_{i} \forall i \in[d]\right\} \sqrt{2} \operatorname{Vol}_{d-2} H \\
& =\sqrt{2} \operatorname{Prob}_{\rho}[E(u)] \operatorname{Vol}_{d-2} H .
\end{aligned}
$$

We are going to estimate $\mathrm{Vol}_{d-2} H$ using Lemma (3.2). So our target is to bound the product of the $\sqrt{2} \beta_{i}=\frac{2 \delta \sqrt{2}}{|u| a_{i} \sin \gamma}$ that are below 1 .

For this end, fix $u \in \mathbf{P}^{*}$ and fix $\gamma$ and consider $v \in \mathbf{P}(u)$ with angle $\gamma$ between $u$ and $v$. The sequence

$$
\frac{2 \delta \sqrt{2}}{|u| a_{2} \sin \gamma}>\frac{2 \delta \sqrt{2}}{|u| a_{3} \sin \gamma}>\cdots>\frac{2 \delta \sqrt{2}}{|u| a_{d} \sin \gamma}
$$

is decreasing. Its first element is larger than 1 by inequality (1). Let $i=i(v)$ be the largest index $j \in[d]$ with $\frac{2 \delta \sqrt{2}}{|u| a_{j} \sin \gamma}>1$. We classify the vectors in $v \in \mathbf{P}(u)$ according to $i(v)$ : define

$$
\mathbf{P}(u)_{j}=\{v \in \mathbf{P}(u): i(v)=j\} .
$$

Now we can use the previous estimate for $\operatorname{Prob}_{\rho}[E(u) \cap E(v)]$ :

$$
\begin{aligned}
\sum_{v \in \mathbf{P}(u)_{j}} \operatorname{Prob}_{\rho}[E(u) \cap E(v)] \leq & \sqrt{2} \operatorname{Prob}_{\rho}[E(u)] \sum_{v \in \mathbf{P}(u)_{j}} \operatorname{Vol}_{d-2} H \\
& \ll \operatorname{Prob}_{\rho}[E(u)] \sum_{v \in \mathbf{P}(u)_{j}} \prod_{i=j+1}^{d}\left(|u| a_{i} \sin \gamma\right)^{-1} \\
& =\operatorname{Prob}_{\rho}[E(u)] \sum_{v \in \mathbf{P}(u)_{j}} \frac{1}{(|u| \sin \gamma)^{d-j} a_{j+1} \ldots a_{d}} .
\end{aligned}
$$

For simpler writing set $\gamma_{j}=\arcsin \frac{2 \delta \sqrt{2}}{|u| a_{j}}$ for $j \in[d]$ and $\gamma_{d+1}=0$ and $U=\left(2.1 a_{1}\right)^{-1}$. The sum over $v \in \mathbf{P}(u)_{j}$ can be estimated from above by the integral (we omit the routine 
details) over all $x \in \mathbf{R}^{d}$ satisfying $|u| \leq|x| \leq U$ such that the angle between vectors $x$ and $u$ lies in $\left[\gamma_{j+1}, \gamma_{j}\right]$. So we have

$$
\begin{aligned}
\sum_{v \in \mathbf{P}(u)_{j}} \frac{1}{(|u| \sin \gamma)^{d-j} a_{j+1} \ldots a_{d}} \ll & \int_{|u|}^{U} \int_{\gamma_{j+1}}^{\gamma_{j}} \frac{r^{d-1}(\sin \gamma)^{d-2} d \gamma d r}{(|u| \sin \gamma)^{d-j} a_{j+1} \ldots a_{d}} \\
& \ll \frac{U^{d}-|u|^{d}}{|u|^{d-j} a_{j+1} \ldots a_{d}} \int_{\gamma_{j+1}}^{\gamma_{j}}(\sin \gamma)^{j-2} d \gamma \\
& \ll \frac{U^{d}}{|u|^{d-j} a_{j+1} \ldots a_{d}} \frac{1}{j-1}\left[\left(\frac{2 \delta \sqrt{2}}{|u| a_{j}}\right)^{j-1}-\left(\frac{2 \delta \sqrt{2}}{|u| a_{j+1}}\right)^{j-1}\right] \\
& \ll \frac{U^{d}}{|u|^{d-1} a_{j}^{j-1} a_{j+1} \ldots a_{d}} \ll \frac{U^{d}}{|u|^{d-1} a_{2} a_{3} \ldots a_{d}} .
\end{aligned}
$$

Here the integral of $(\sin \gamma)^{j-2}$ is estimated by substituting $t=\sin \gamma$ and ignoring the $\left(1-t^{2}\right)^{-1 / 2}$ factor, which is bounded since $\sin \gamma<0.64$. Recall that $u \in \mathbf{P}^{*}$ implies that $\frac{1}{a_{1}|u|} \in[2.1,2.3]$. Adding the above inequalities for $j=2,3, \ldots, d$ we get that

$$
\begin{aligned}
\sum_{j=2}^{d} \sum_{v \in \mathbf{P}(u)_{j}} \operatorname{Prob}_{\rho}[E(u) \cap E(v)] & \ll \operatorname{Prob}_{\rho}[E(u)] \frac{U^{d}}{|u|^{d-1} a_{2} a_{3} \ldots a_{d}} \\
& \ll \operatorname{Prob}_{\rho}[E(u)] \frac{U}{a_{2} a_{3} \ldots a_{d}} \\
& \ll \frac{1}{V} \operatorname{Prob}_{\rho}[E(u)],
\end{aligned}
$$

since $U /|u| \leq 2.3 / 2.1$ and $U=\left(2.1 a_{1}\right)^{-1}$. So we have, replacing the implicit constant in $\ll$ by the explicit constant $c=c(d)$,

$$
\sum_{j=2}^{d} \sum_{v \in \mathbf{P}(u)_{j}} \operatorname{Prob}_{\rho}[E(u) \cap E(v)] \leq \frac{c}{V} \operatorname{Prob}_{\rho}[E(u)] \leq \frac{1}{2} \operatorname{Prob}_{\rho}[E(u)],
$$

since $c / V$ becomes smaller than $1 / 2$ if $V$ is large enough.

We can finish the proof now. For large enough $V$ we have

$$
\begin{aligned}
\operatorname{Prob}_{\rho}\left[\bigcup_{u \in \mathbf{P}} E(u)\right] & \geq \sum_{u \in \mathbf{P}^{*}}\left(\operatorname{Prob}_{\rho}[E(u)]-\sum_{v \in \mathbf{P}(u)} \operatorname{Prob}_{\rho}[E(u) \cap E(v)]\right) \\
& \geq \frac{1}{2} \sum_{u \in \mathbf{P}^{*}} \operatorname{Prob}_{\rho}[E(u)] \gg \frac{1}{V} .
\end{aligned}
$$

\section{ACKNOWLEDGMENTS}

I am indebted to two anonymous referees (especially the second one) for useful comments and remarks. I hope their suggestions have made the paper more readable. Support from Hungarian National Foundation Grants T 046246 and T 037846 is also acknowledged. 


\section{REFERENCES}

[1] I. Bárány, Intrinsic volumes and $f$-vectors of random polytopes, Math Annalen 285 (1989), 671-699.

[2] I. Bárány and J. Matoušek, On randomized integer convex hull, Discrete Comp Geom 32 (2005), 135-142.

[3] I. Bárány and D. G. Larman, Convex bodies, economic cap coverings, random polytopes, Mathematika 35 (1998), 274-291.

[4] I. Bárány and N. Tokushige, The minimum area of convex lattice $n$-gons, Combinatorica 24 (2004), 171-185.

[5] L. Danzer, B. Grünbaum, and V. Klee, "Helly's theorem and its relatives," Proc Symp Pure Math, Vol VIII, Convexity, Am Math Soc, Providence, RI, 1963.

[6] G. Ewald, D. G. Larman, and C. A. Rogers, The directions of the line segments and of the $r$-dimensional balls on the boundary of a convex body in Euclidean space, Mathematika 17 (1970), 1-20.

[7] C. H. Hardy and E. M. Wright, An introduction to the theory of numbers, Clarendon Press, Oxford, 1960.

[8] R. Kannan and L. Lovász, Covering minima and lattice point free convex bodies, Ann Math 128 (1988), 577-622.

[9] A. Khintchin, A quantitative formulation of Kronecker's theory of approximation (in Russian), Izv Akad Nauk SSSR Mat 12 (1948), 113-122.

[10] A. M. Macbeath, A theorem on non-homogeneous lattices, Ann Math 56 (1952), 269-293. 